\title{
ANTS 2016 special issue: Editorial
}

\author{
Marco Dorigo ${ }^{1}$ (D) $\cdot$ Mauro Birattari ${ }^{1} \cdot{\text { Xiaodong } \text { Li }^{2} \cdot \text { Manuel López-Ibáñez }}^{3} \cdot$ \\ Kazuhiro Ohkura ${ }^{4}$. Carlo Pinciroli ${ }^{5}$. Thomas Stützle ${ }^{1}$
}

Received: 24 October 2017 / Accepted: 4 November 2017 / Published online: 13 November 2017

(C) Springer Science+Business Media, LLC, part of Springer Nature 2017

This special issue of the Swarm Intelligence journal is dedicated to the publication of extended versions of some of the best papers presented at ANTS 2016, Tenth International Conference on Swarm Intelligence, which took place in Brussels on September 7-9, 2016.

The ANTS series of conferences has taken place at the Université Libre de Bruxelles, Brussels, Belgium, every other year since 1998. Since 2010 (i.e., starting with the seventh edition of the conference), the authors of the contributions accepted as full papers at the conference were invited to submit an extended version of their work for possible inclusion in a dedicated special issue of the Swarm Intelligence journal.

Marco Dorigo

mdorigo@ulb.ac.be

Mauro Birattari

mbiro@ulb.ac.be

Xiaodong Li

xiaodong.li@rmit.edu.au

Manuel López-Ibáñez

manuel.lopez-ibanez@manchester.ac.uk

Kazuhiro Ohkura

kohkura@hiroshima-u.ac.jp

Carlo Pinciroli

cpinciroli@wpi.edu

Thomas Stützle

stuetzle@ulb.ac.be

1 IRIDIA, Université Libre de Bruxelles, Brussels, Belgium

2 RMIT University, Melbourne, Australia

3 University of Manchester, Manchester, UK

4 Hiroshima University, Hiroshima, Japan

5 Worcester Polytechnic Institute, Worcester, MA, USA 
Six of the article submitted to the 2016 edition of the conference were accepted for publication after at least two rounds of reviews with comments by at least three referees.

The special issue opens with "Cooperative object transport with a swarm of e-puck robots: robustness and scalability of evolved collective strategies." In this paper, Muhanad H. Mohammed Alkilabi, Aparajit Narayan, and Elio Tuci show how coordinated collective transport can be obtained with robots with minimalistic capabilities. The robots involved in the study are assumed capable of pushing (not pulling) an object, detecting the object (using a camera and a ring of proximity sensors), and estimating their displacement (using a custom-made optical flow sensor). The robot controller is a neural network configured through artificial evolution in simulated experiments and later validated with e-puck robots. The authors evaluate the scalability and generality of the behavior and also provide insight on its internal logic. The minimalist assumptions in this work could be used as a basis to evaluate future implementations of collective transport systems and to assess the costs and benefits of using increasingly capable robots.

In "Learning cluster-based classification systems with ant colony optimization algorithms," Khalid M. Salama and Ashraf M. Abdelbar introduce a classification algorithm that first determines a clustering of the whole data set into data subsets. Subsequently, the algorithm uses local classification models, each learned separately for a data subset, to perform the classification task of new data instances. The data subsets are determined using an ACO-based clustering approach, while for each data subset a classification algorithm such as decision trees, rule-based systems, or support vector machines is used. An ensemble approach is introduced that can give a weighted combination of the local classifications to further improve performance. The experimental results obtained on a large number of data sets indicate that the highest predictive accuracy is obtained by a system configuration that allows different classifiers to be used for different data subsets and that employs the ensemble-based classification approach.

In "Automatic synthesis of rulesets for programmable stochastic self-assembly of rotationally symmetric robotic modules," Bahar Haghighat and Alcherio Martinoli tackle the problem of generating rulesets for programmable self-assembling robots. Extending previous work on graph grammars, the authors propose two novel algorithms to synthesize sequential and concurrent rulesets. The novelty of these algorithms is twofold: first, the number of rules increases linearly with the number of latches available on the robots, rather than following a square law; second, the generated ruleset can be used on real robots without modification, in contrast to rulesets generated with previous approaches. The algorithms are evaluated using both real Lily robots and two different simulation frameworks, which highlight different aspects of the dynamics of the system. This work is a step forward in the principled design of modular systems for medical and space applications, where stochastic self-assembly will be an important part of their dynamics.

In "Continuous time gathering of agents with limited visibility and bearing-only sensing," Levi Itzhak Bellaiche and Alfred Bruckstein study the aggregation of a swarm of simple point agents in a two-dimensional space. In particular, the authors focus on agents whose capabilities are severely limited: each agent can only sense the direction to the neighboring peers that are located within a finite sensing range. The contribution made in the article is to prove that, by following a simple behavioral rule, these agents gather to a common meeting point in finite time. Indeed, the distance between each pair of agents that initially see each other decreases monotonically in time.

In "Stochastic stability of particle swarm optimization," Adam Erskine, Thomas Joyce, and J. Michael Herrmann examine the dynamics of particle swarm optimization (PSO) in the framework of random dynamical systems. With such an approach, the swarm dynamics 
are quasi-linear, which allows an analytical treatment of their stability. The simplification assumption on the stochasticity of the PSO algorithm is no longer required. Instead, a stochastically exact formulation can be used to show that the range of stable parameters is actually larger than the one estimated by previous studies on PSO dynamics. This study also facilitates an explanation on the differences and similarities between theoretical and empirical results for PSO.

In "PolyACO+: a multi-level polygon-based ant colony optimization classifier," Morten Goodwin, Torry Tufteland, Guro Ødesneltvedt, and Anis Yazidi introduce PolyACO+, an improved version of PolyACO, which is a previously proposed classification algorithm based on ant colony optimization. The main improvements over PolyACO are that PolyACO+ is able to classify multiple classes and to handle more than two features. In the article, the authors empirically show that, thanks to the proposed improvements, the performance of PolyACO + is comparable with the one of state-of-the-art classification techniques such as SVM, neural networks, and AntMiner+.

The high quality of the six papers contained in this special issue is the result of the collaboration of a large number of people: the authors, who submitted their very best work to the journal; the referees, who helped in the selection of the published papers; and finally, the many people at Springer who assisted us in the production phase. We thank them all for their help to make this special issue possible.

October 2017

Marco Dorigo

Mauro Birattari

Xiaodong Li

Manuel López-Ibáñez

Kazuhiro Ohkura

Carlo Pinciroli

Thomas Stuitzle 